\title{
Resonance asymptotics for quantum waveguides with semitransparent multi-perforated wall
}

\author{
A. M. Vorobiev \\ ITMO University, Kronverkskiy, 49, St. Petersburg, 197101, Russia \\ lenden31@yandex.ru
}

DOI 10.17586/2220-8054-2021-12-4-462-471

\begin{abstract}
A pair of coupled quantum waveguides with a common semitransparent wall is considered. The wall has a finite number of small windows. We consider resonance states localized near each window. The presence of several windows forces one to describe their common influence differently from that of the single-window case. Using the "matching of asymptotic expansions" method, we derive formulas for resonances and resonance states.
\end{abstract}

Keywords: resonance, resonance state, waveguide, asymptotics, semitransparency.

Received: 5 June 2021

Revised: 12 August 2021

\section{Introduction}

There is a large class of mathematical and physical problems concerning to "systems with small coupling windows", i.e., systems of connected quantum waveguides, Helmholtz resonators and other structures with perturbation caused by small windows on different boundaries. Such systems have long been of interest to physicists and mathematicians. Transport properties of waveguides and other additional phenomena caused by resonators are widely used in electrodynamics and theories of acoustic and electromagnetic waves.

Currently, scientists study such systems in the context of nanoelectronics. The development in this field has led to the creation of a new class of objects used in electronic devices - quantum threads (nanowhiskers), quantum dots, antidots and so on. Of course, "quantum" is a keyword here because studying mesoscopic systems (systems where the coherence of the phases of the electrons is preserved on a scale much larger than atomic) is absolutely impossible if we ignore quantum properties of the electron. Obviously, taking into account the quantum behavior of an electron requires the development of fundamentally new physical, and, most importantly, mathematical approaches.

When we consider mesoscopic systems, we actually mean studying wave propagation in waveguides and other structures. Properties of this propagation are described as spectral properties of corresponding mathematical operator. This is usually a Schrödinger operator. Laplace operator is also applicable for problems considering the ballistic mode. Resonance phenomena are widely used for development of new nanoelectronic devices.

Systems with small coupling windows were studied since the beginning of the 20th century [1,2]. Currently, we use term "asymptotic analysis" for large class of problems including resonance phenomena. Great variety of electronic device caused great variety of systems in resonance problems. Different combinations of waveguides and resonators were considered [3-6], in addition to different geometry characteristics [7, 8], windows amount [9-11], and boundary conditions $[12,13]$.

The core of the mathematical approach in this work is "Matching asymptotic expansions of solutions of boundary problems". This method was previously described in [14]. This method is intended for boundary problems of equations containing naturally occurring small parameter. It is very typical for mathematical physics problems, in particular for problems we described previously.

Semitransparency was studied by Ikebe, Shimada, Exner, Kreicirik, Popov in [15-20] in terms of spectral and scattering properties. Resonance asymptotics for such systems were studies in [21,22].

\section{Preliminaries}

Let us consider the pair of quantum waveguides in two-dimensional Cartesian coordinates with widths $d_{-}$ and $d_{+}$

The common wall of both waveguides is semitransparent and is coupled through small windows. Semitransparency was studied in [21] for the same system but with single window. In this work, we consider finite number of windows. We will rely on the results of that work but we won't describe in detail the preliminary calculations. So let's take a quick look at used formulas and assumptions. 


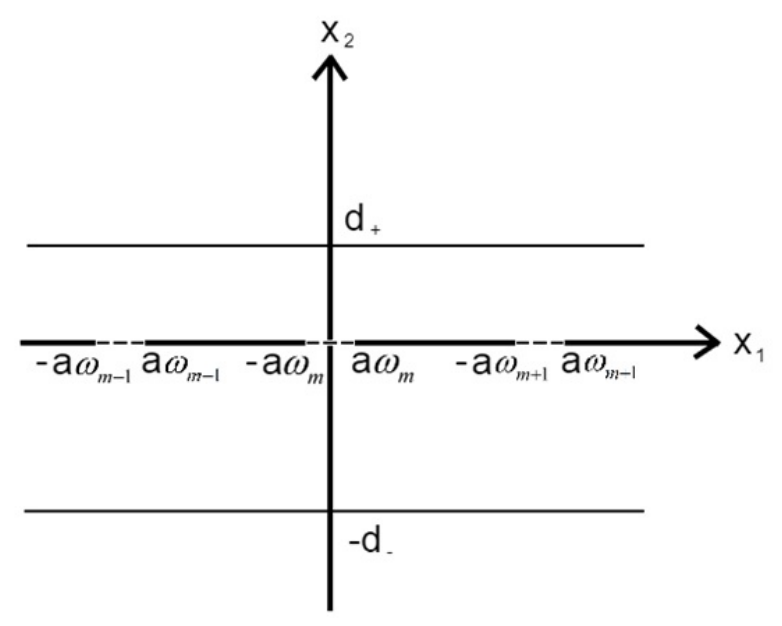

FIG. 1. Waveguides with common semitransparent wall (windows abscissas are relative to each center)

The wall semitransparency is described by the parameter $\alpha$. Generally, $\alpha \in(0 ;+\infty)$, where zero value means no barrier and infinity means absolutely nontransparent barrier. Zero value is not included because actually it is not the case of the considered problem.

Boundary conditions on the walls are the key point: we choose the Dirichlet conditions on non-common walls but semitransparency causes special requirements for common wall. When a wave passes through the barrier, a jump occurs in the derivative of the considered function $u\left(x_{1}, x_{2}\right)$, so, we will consider specific boundary conditions:

$$
\left\{\begin{array}{l}
u_{+}=u_{-} \\
u_{+}^{\prime}-u_{-}^{\prime}=\alpha u
\end{array}\right.
$$

where $u_{+-}^{\prime}$ are vertical derivatives at the top and bottom of the wall. The conditions of such type appear if one considers the singular potential supported on hypersurface. These potentials have been intensively investigated during last two decades (see, e.g., [23-25]).

Problems with several windows may be very different. The number of windows can be finite or infinite; the "order of smallness" can vary for each window. We consider somewhat simple case - finite number of windows with the same order of smallness.

Let us have $n$ coupling windows with the centers at the points $\left(x_{q} ; 0\right)$, where $q=1 . . n$. Each window has width $2 a_{q}=2 a--\omega_{m} \omega_{m-1} \omega_{m+1}$. Parameter $a$ is exactly common order of smallness. We will construct asymptotics in $a$. This also means that $\omega_{q}, q=1 . . n$, describes the relative sizes of all the windows.

Unperturbed eigenfunctions of the transversal problem for the Laplace operator with semitransparency can be written as follows:

$$
\chi_{n}(x)=\left\{\begin{array}{l}
-C_{n} \sin \left(d_{-} \nu_{n}\right) \sin \left(\left(x_{2}-d_{+}\right) \nu_{n}\right), x_{2}>0, \\
C_{n} \sin \left(d_{+} \nu_{n}\right) \sin \left(\left(x_{2}+d_{-}\right) \nu_{n}\right), x_{2}<0 .
\end{array}\right.
$$

One can see that it satisfies conditions (1). The corresponding eigenvalues are denoted as $\nu_{n}$ and can be found from the equation $-\nu \operatorname{ctg}\left(d_{+} \nu\right)-\nu \operatorname{ctg}\left(d_{-} \nu\right)=\alpha$. For investigating resonances, we need to choose "threshold" value. Threshold is such value of $\nu_{n}$, that there are no summands with imaginary part exponent in Green's function series which are lower than $\nu_{n}$. Imaginary exponent corresponds to periodic summands which mean propagating waves. We will seek terms of asymptotic expansions close to the second threshold $\nu_{2}$.

"Perturbed" eigenvalue, also known as quasi-eigenfrequency, will be denoted as $k_{a}$. Difference between $k_{a}$ and $\nu_{2}$ is actually small value. We will use convenient expansion for its asymptotics:

$$
\sqrt{\nu_{2}^{2}-k_{a}^{2}}=\sum_{j=2}^{\infty} \sum_{i=0}^{[j / 2]-1} k_{j i} a^{j} \ln ^{i} \frac{a}{a_{0}} .
$$

The Green function for waveguide is well-known [25] and appears as follows in our case:

$$
G^{ \pm}(x, y, k)=\sum_{n=1}^{\infty} \frac{\chi_{n}\left(x_{2}\right) \cdot \chi_{n}\left(y_{2}\right)}{2 p_{n}} \cdot e^{-p_{n}\left|x_{1}-y_{1}\right|}, \text { where } p_{n}^{ \pm}=p_{n}=\sqrt{\nu_{n}^{2}-k_{a}^{2}} .
$$


Finally, we can write down eigenfunctions for perturbed case near each window:

$$
\left\{\begin{array}{l}
\psi_{a}(x)=\left.\sqrt{\nu_{2}^{2}-k_{a}^{2}} \cdot \sum_{j=0}^{\infty} \sum_{q=1}^{n} a^{j} P_{j+1}^{q}\left(D_{y}, \ln \frac{a}{a_{0}}\right) G^{+}(x, y, k)\right|_{y=\left(x_{q} ; 0\right)}, x \in \Omega^{+} \backslash \bigcup_{q=1}^{n} S_{a_{0}\left(a / a_{0}\right)^{1 / 2}}^{q}, \\
\psi_{a}(x)=\sum_{j=1}^{\infty} \sum_{i=0}^{[(j-1) / 2]} v_{j i}\left(\frac{x}{a}\right) a^{j} \ln ^{i} \frac{a}{a_{0}}, x \in \bigcup_{q=1}^{n} S_{2 a_{0}\left(a / a_{0}\right)^{1 / 2}}^{q}, \\
\psi_{a}(x)=-\left.\sqrt{\nu_{2}^{2}-k_{a}^{2}} \cdot \sum_{j=0}^{\infty} \sum_{q=1}^{n} a^{j} P_{j+1}^{q}\left(D_{y}, \ln \frac{a}{a_{0}}\right) G^{-}(x, y, k)\right|_{y=\left(x_{q} ; 0\right)}, x \in \Omega^{-} \backslash \bigcup_{q=1}^{n} S_{a_{0}\left(a / a_{0}\right)^{1 / 2}}^{q} .
\end{array}\right.
$$

Here, $S_{a_{0}\left(a / a_{0}\right)^{1 / 2}}^{q}$ is as sphere with a center at $q$-th window. Last important thing is differential operator $P_{j}^{q}$. It is described as follows:

$$
\begin{gathered}
P_{1}\left(D_{y}, \ln \frac{a}{a_{0}}\right)=a_{10}^{(1)} D_{y}^{1}, \quad D_{y}^{n}=\frac{\partial^{n}}{\partial n_{y}^{n}}, \quad P_{m}\left(D_{y}, \ln \frac{a}{a_{0}}\right)=\sum_{j=1}^{m-1} \sum_{i=0}^{[(j-1) / 2]} a_{j i}^{(m)}\left(\ln \frac{a}{a_{0}}\right)^{i} D_{y}^{m-j+1}, \quad m \geq 2, \\
P_{m}^{q}\left(D_{y}, \ln \frac{a}{a_{0}}\right)=\alpha_{q} P_{m}\left(D_{y}, \ln \frac{a}{a_{0}}\right)
\end{gathered}
$$

Here, $\alpha_{q}$ are very important coefficients which describe operator action in distribution between windows.

\section{Calculating}

Boundary problems for $v_{j i}\left(\frac{x}{a}\right)$ from (4) can be obtained in the following manner. We substitute the series (4) and (3) into the Helmholtz equation (for $k=k_{a}$ ) and then change variables $\xi=\frac{x}{a}$. The coefficients in the terms with the same powers of $a$ and $\ln \frac{a}{a_{0}}$ should be equal. Hence, we obtain the following problems:

$$
\Delta_{\xi} v_{j i}=-\sum_{p=0}^{j-3} \sum_{q=0}^{[p / 2]} \Lambda_{p q} v_{j-p-2, i-q}, \quad \xi \in R^{2} \backslash \gamma, \quad v_{j i}=0, \xi \in \gamma
$$

where $\gamma=\left\{\xi \mid \xi_{2}=0 \wedge \xi_{1} \in(-\infty ;-1] \cup[1 ;+\infty)\right\}$ and $\Lambda_{p q}$ are the coefficients of the series:

$$
k_{a}^{2}=\sum_{p} \sum_{q} \Lambda_{p q} a^{p} \ln ^{q} \frac{a}{a_{0}} .
$$

As next step we need to introduce operator $M_{p q}(U)$ - it changes variables in expressions $\mathrm{U}\left(\xi=\frac{x}{a}, \ln r=\ln \rho+\ln a\right)$ and filters summand with $a^{p} \ln ^{q} \frac{a}{a_{0}} \varphi(\xi)$. Also, $M_{p}=\sum_{q} M_{p q}$, it is used to obtain all summands with $a^{p}$.

\subsection{Calculating of $\mathbf{k}_{\mathbf{2 0}}$}

Considering matching at $q$-th window we can obtain the following $a$-degree selections:

$$
\begin{aligned}
& a^{-1} M_{1}\left( \pm\left.\sqrt{\nu_{2}^{2}-k_{a}^{2}} \cdot \sum_{q=1}^{n} P_{1}^{q} G^{ \pm}\left(x, y, k_{a}\right)\right|_{y=\left(x_{q} ; 0\right)}\right)= \\
& \pm \frac{a_{10}^{(1)} C_{2}^{2} \nu_{2}^{2} \sin ^{2}\left(d_{\mp} \nu_{2}\right) \cos ^{2}\left(d_{ \pm} \nu_{2}\right)}{2} \cdot \sum_{p=1}^{n} \alpha_{p} \rho \sin \theta \mp \frac{1}{\pi} \alpha_{q} k_{20} a_{10}^{(1)} \rho^{-1} \sin \theta .
\end{aligned}
$$

An extremely important point to note $-k$-singularity has its place in all window summands but $x$-singularity exists only in current window, so we have $\sum_{p=1}^{n} \alpha_{p}$ for positive $\rho$ degree and just $\alpha_{q}$ for negative degree being in context of $q$-th window.

In accordance with the lemma concerning the $v_{j i}$ representation in the form of a linear combination of harmonic functions, we need to choose those functions as a summary of all windows. Function $f_{1}(z)=\frac{1}{2}\left(z+\sqrt{z^{2}-1}\right)$ was 
used for single window so we choose summary function as follows:

$$
\begin{aligned}
& \widehat{f}_{1}(z)=\sum_{q=1}^{n} \alpha_{q} \omega_{q} f_{1}\left(\frac{z-x_{q}}{\omega_{q}}\right)= \\
& \left\{\begin{array}{l}
\sum_{q=1}^{n} \alpha_{q} \omega_{q} \frac{z-x_{q}}{\omega_{q}}-\sum_{q=1}^{n} \alpha_{q} \frac{\omega_{q}^{2}}{4 z}-\sum_{q=1}^{n} \alpha_{q} \frac{\omega_{q}^{2} x_{q}}{4 z^{2}}-\sum_{q=1}^{n} \alpha_{q}\left(\frac{\omega_{q}^{2} x_{q}^{2}}{4}+\frac{\omega_{q}^{4}}{16}\right) \frac{1}{z^{3}}-\ldots, z \rightarrow+\infty \\
\sum_{q=1}^{n} \alpha_{q} \frac{\omega_{q}^{2}}{4 z}+\sum_{q=1}^{n} \alpha_{q} \frac{\omega_{q}^{2} x_{q}}{4 z^{2}}+\sum_{q=1}^{n} \alpha_{q}\left(\frac{\omega_{q}^{2} x_{q}^{2}}{4}+\frac{\omega_{q}^{4}}{16}\right) \frac{1}{z^{3}}+\ldots, z \rightarrow-\infty
\end{array}\right.
\end{aligned}
$$

For different coordinates, we will use notation $\widehat{f}_{i}(z)=\widehat{X}_{i}(z)+i \widehat{Y}_{i}(z)$. To match terms increasing on $\rho \rightarrow \infty$ in accordance with (6), we shall select $v_{10}(\xi)$ in such a way:

$$
v_{10}(\xi)=\frac{a_{10}^{(1)} C_{2}^{2} \nu_{2}^{2} \sin ^{2}\left(d_{-} \nu_{2}\right) \cos ^{2}\left(d_{+} \nu_{2}\right)}{2} \cdot \widehat{Y_{1}}(\xi)+\frac{a_{10}^{(1)} C_{2}^{2} \nu_{2}^{2} \sin ^{2}\left(d_{+} \nu_{2}\right) \cos ^{2}\left(d_{-} \nu_{2}\right)}{2} \cdot \widehat{Y_{1}}\left(\xi^{*}\right) .
$$

Hence, we can match terms of order $\rho^{-1} \sin \theta$ for each window and get the system of equations:

$$
\begin{gathered}
\mp \frac{1}{\pi} \alpha_{q} k_{20} a_{10}^{(1)}= \pm \frac{a_{10}^{(1)} C_{2}^{2} \nu_{2}^{2}\left(\sin ^{2}\left(d_{-} \nu_{2}\right) \cos ^{2}\left(d_{+} \nu_{2}\right)+\sin ^{2}\left(d_{+} \nu_{2}\right) \cos ^{2}\left(d_{-} \nu_{2}\right)\right)}{2} \cdot\left(\sum_{p=1}^{n} \alpha_{p} \frac{\omega_{p}^{2}}{4}\right) \Rightarrow \\
\sum_{p=1}^{n} \alpha_{p} c_{\omega_{p}}=\beta \alpha_{q}, \beta=\frac{-2 k_{20}}{\pi C_{2}^{2} \nu_{2}^{2}\left[\sin ^{2}\left(d_{-} \nu_{2}\right) \cos ^{2}\left(d_{+} \nu_{2}\right)+\sin ^{2}\left(d_{+} \nu_{2}\right) \cos ^{2}\left(d_{-} \nu_{2}\right)\right]} \\
=\frac{-4 k_{20}}{\pi C_{2}^{2} \nu_{2}^{2}\left[1-\cos \left(2 d_{-} \nu_{2}\right) \cos \left(2 d_{+} \nu_{2}\right)\right]} .
\end{gathered}
$$

This system has nontrivial solution if:

$$
\operatorname{det}\left(\begin{array}{llll}
c_{\omega_{1}}-\beta & c_{\omega_{1}} & \ldots & c_{\omega_{1}} \\
c_{\omega_{2}} & c_{\omega_{2}}-\beta & \ldots & c_{\omega_{2}} \\
\ldots & \ldots & \ldots & \ldots \\
c_{\omega_{n}} & c_{\omega_{n}} & \ldots & c_{\omega_{n}}-\beta
\end{array}\right)=0
$$

where $\frac{\omega_{q}^{2}}{4}$ is actually harmonic capacity of the corresponding segment in $R^{2}$, so we will use sometimes a symbol: $c_{\omega_{q}}=\frac{\omega_{q}^{2}}{4}$. Determinant of this matrix is $(-\beta)^{n-1}\left(\left(\sum_{q=1}^{n} c_{\omega_{q}}\right)-\beta\right)$. Hence:

$$
\begin{gathered}
\beta=\sum_{q=1}^{n} c_{\omega_{q}} \Rightarrow \frac{-4 k_{20}}{\pi C_{2}^{2} \nu_{2}^{2}\left[1-\cos \left(2 d_{-} \nu_{2}\right) \cos \left(2 d_{+} \nu_{2}\right)\right]}=\sum_{q=1}^{n} c_{\omega_{q}} \Rightarrow \\
k_{20}=-\frac{\pi C_{2}^{2} \nu_{2}^{2}}{4}\left[1-\cos \left(2 d_{-} \nu_{2}\right) \cos \left(2 d_{+} \nu_{2}\right)\right] \sum_{q=1}^{n} c_{\omega_{q}} .
\end{gathered}
$$

If you compare it with $k_{20}$ for the single window case, you will see that the only difference is sum $\sum_{q=1}^{n} c_{\omega_{q}}$. It is actually something like "common size" of the windows, characterized by sum of capacities. For $n=1$ it equals $\frac{1}{4}$.

\subsection{Calculating of $\mathbf{k}_{\mathbf{3 0}}$}

Procedure for $k_{30}$ is absolutely the same. Selecting of $a^{2}$ gives us:

$$
\begin{aligned}
& \pm a^{-2} M_{2}\left(\left.\sqrt{\nu_{2}^{2}-k_{a}^{2}} \cdot \sum_{q=1}^{n} P_{1}^{q} G^{ \pm}\left(x, y, k_{a}\right)\right|_{y=\left(x_{q} ; 0\right)}\right)= \\
& \frac{-a_{10}^{(1)} C_{2}^{2} \sin ^{2}\left(d_{\mp} \nu\right) \nu_{2}^{3} \cos \left(d_{ \pm} \nu_{2}\right) \sin \left(d_{ \pm} \nu_{2}\right)}{8} \sum_{p=1}^{n} \alpha_{p} \rho^{2} \cos 2 \theta \mp \frac{1}{\pi} \alpha_{q} k_{30} a_{10}^{(1)} \rho^{-1} \sin (\theta),
\end{aligned}
$$




$$
\begin{aligned}
& \pm a^{-2} M_{2}\left(\left.\sqrt{\nu_{2}^{2}-k_{a}^{2}} \cdot \sum_{q=1}^{n} a \cdot P_{2}^{q} G^{ \pm}\left(x, y, k_{a}\right)\right|_{y=\left(x_{q} ; 0\right)}\right)= \\
& \frac{a_{10}^{(2)} C_{2}^{2} \sin ^{2}\left(d_{\mp} \nu_{2}\right) \nu_{2}^{3} \cdot \sin \left(d_{ \pm} \nu_{2}\right) \cos \left(d_{ \pm} \nu_{2}\right)}{2} \sum_{p=1}^{n} \alpha_{p} \rho \sin \theta \mp \frac{1}{\pi} \alpha_{q} k_{30} a_{10}^{(2)} \rho^{-2} \cos 2 \theta .
\end{aligned}
$$

Harmonic function of the second order uses the same formula except one more multiplier $\omega_{q}$ and uses $f_{2}(z)=$ $z \cdot f_{1}(z)=\frac{1}{2}\left(z^{2}+z \sqrt{z^{2}-1}\right):$

$$
\begin{aligned}
& \widehat{f}_{2}(z)=\sum_{q=1}^{n} \alpha_{q} \omega_{q}^{2} f_{2}\left(\frac{z-x_{q}}{\omega_{q}}\right)= \\
& \left\{\begin{array}{l}
\sum_{q=1}^{n} \alpha_{q} \omega_{q}^{2} \frac{z^{2}}{\omega_{q}^{2}}-\sum_{q=1}^{n} \alpha_{q} \omega_{q}^{2} \frac{2 x_{q} z}{\omega_{q}^{2}}-\sum_{q=1}^{n} \alpha_{q} \omega_{q}^{2} \frac{\omega_{q}^{2}}{16 z^{2}}-\sum_{q=1}^{n} \alpha_{q} \omega_{q}^{2} \frac{2 \omega_{q}^{2} x_{q}}{16 z^{3}}-\ldots, z \rightarrow+\infty \\
\sum_{q=1}^{n} \alpha_{q} \omega_{q}^{2} \frac{\omega_{q}^{2}}{16 z^{2}}+\sum_{q=1}^{n} \alpha_{q} \omega_{q}^{2} \frac{2 \omega_{q}^{2} x_{q}}{16 z^{3}}+\ldots, z \rightarrow-\infty
\end{array}\right.
\end{aligned}
$$

Unfortunately, it contains not only positive $\rho$ degree, so we need to subtract corresponding additional summand using $\widehat{X_{1}}(\xi)$ :

$$
\begin{aligned}
& v_{20}(\xi)=\frac{-a_{10}^{(1)} C_{2}^{2} \sin ^{2}\left(d_{-} \nu\right) \nu_{2}^{3} \cos \left(d_{+} \nu_{2}\right) \sin \left(d_{+} \nu_{2}\right)}{8} \widehat{X}_{2}(\xi) \\
& \frac{-a_{10}^{(1)} C_{2}^{2} \sin ^{2}\left(d_{+} \nu\right) \nu_{2}^{3} \cos \left(d_{-} \nu_{2}\right) \sin \left(d_{-} \nu_{2}\right)}{8} \widehat{X}_{2}\left(\xi^{*}\right) \\
& \frac{-2 a_{10}^{(1)} C_{2}^{2} \sin ^{2}\left(d_{-} \nu\right) \nu_{2}^{3} \cos \left(d_{+} \nu_{2}\right) \sin \left(d_{+} \nu_{2}\right)}{8} \frac{\sum_{q=1}^{n} \alpha_{q} x_{q}}{\sum_{q=1}^{n} \alpha_{q}} \widehat{X_{1}}(\xi) \\
& \frac{-2 a_{10}^{(1)} C_{2}^{2} \sin ^{2}\left(d_{+} \nu\right) \nu_{2}^{3} \cos \left(d_{-} \nu_{2}\right) \sin \left(d_{-} \nu_{2}\right)}{8} \frac{\sum_{q=1}^{n} \alpha_{q} x_{q}}{\sum_{q=1}^{n} \alpha_{q}} \widehat{X_{1}}\left(\xi^{*}\right) \\
& +\frac{a_{10}^{(2)} C_{2}^{2} \sin ^{2}\left(d_{-} \nu_{2}\right) \nu_{2}^{3} \cdot \sin \left(d_{+} \nu_{2}\right) \cos \left(d_{+} \nu_{2}\right)}{2} \widehat{Y_{1}}(\xi) \\
& -\frac{a_{10}^{(2)} C_{2}^{2} \sin ^{2}\left(d_{+} \nu_{2}\right) \nu_{2}^{3} \cdot \sin \left(d_{-} \nu_{2}\right) \cos \left(d_{-} \nu_{2}\right)}{2} \widehat{Y_{1}}\left(\xi^{*}\right) .
\end{aligned}
$$

Equations system is similar to previous:

$$
\begin{aligned}
& \mp \frac{1}{\pi} \alpha_{q} k_{30} a_{10}^{(1)}= \pm \frac{a_{10}^{(2)} C_{2}^{2} \sin ^{2}\left(d_{-} \nu_{2}\right) \nu_{2}^{3} \cdot \sin \left(d_{+} \nu_{2}\right) \cos \left(d_{+} \nu_{2}\right)}{2}\left(\sum_{p=1}^{n} \alpha_{p} \frac{\omega_{p}^{2}}{4}\right) \\
& \mp \frac{a_{10}^{(2)} C_{2}^{2} \sin ^{2}\left(d_{+} \nu_{2}\right) \nu_{2}^{3} \cdot \sin \left(d_{-} \nu_{2}\right) \cos \left(d_{-} \nu_{2}\right)}{2}\left(\sum_{p=1}^{n} \alpha_{p} \frac{\omega_{p}^{2}}{4}\right) \\
& \Rightarrow \sum_{p=1}^{n} \alpha_{p} c_{\omega_{p}}=\beta \alpha_{q}, \beta=\frac{-8 k_{30}}{\pi C_{2}^{2} \nu_{2}^{3}\left[\sin \left(d_{-} \nu_{2}\right) \sin \left(d_{+} \nu_{2}\right) \sin \left(\left(d_{-}-d_{+}\right) \nu_{2}\right)\right]} .
\end{aligned}
$$

Determinant of this matrix is $(-\beta)^{n-1}\left(\left(\sum_{q=1}^{n} c_{\omega_{q}}\right)-\beta\right)$. Hence:

$$
\begin{gathered}
\beta=\sum_{q=1}^{n} c_{\omega_{q}} \Rightarrow \frac{-8 k_{30}}{\pi C_{2}^{2} \nu_{2}^{3}\left(\sin \left(d_{-} \nu_{2}\right) \sin \left(d_{+} \nu_{2}\right) \sin \left(\left(d_{-}-d_{+}\right) \nu_{2}\right)\right)}=\sum_{q=1}^{n} c_{\omega_{q}} \Rightarrow \\
k_{30}=\frac{-\pi C_{2}^{2} \nu_{2}^{3}\left(\sin \left(d_{-} \nu_{2}\right) \sin \left(d_{+} \nu_{2}\right) \sin \left(\left(d_{-}-d_{+}\right) \nu_{2}\right)\right)}{8} \sum_{q=1}^{n} c_{\omega_{q}} .
\end{gathered}
$$




\subsection{Calculating of $\mathbf{k}_{\mathbf{4 0}}$}

As we previously noticed, equation (5) is homogeneous for $v_{10}(\xi), v_{20}(\xi)$, but becomes more complicated for the next step. So we need to solve the Poisson equation:

$$
\Delta_{\xi} v_{30}=-\frac{\pi^{2}}{d_{+}^{2}} v_{10}, \quad v_{30}(\xi)=0, \quad \xi \in \gamma, \quad \gamma=\left\{\xi \mid \xi_{2}=0 \wedge \xi_{1} \in(-\infty ;-1] \cup[1 ;+\infty)\right\} .
$$

The solution of this boundary problem can be presented as:

$$
v_{30}(\xi)=\widehat{v}_{30}(\xi)+\widetilde{v}_{30}(\xi),
$$

where $\widehat{v}_{30}(\xi)$ is solution of homogeneous Laplace equation satisfying the boundary conditions (as we seek for previous steps) and $\widetilde{v}_{30}(\xi)$ is particular solution of inhomogeneous Laplace equation satisfying the boundary conditions. $M$ operator expressions are not much different with single-window ones:

$$
\begin{aligned}
& a^{-3} M_{30}\left( \pm\left.\sqrt{\nu_{2}^{2}-k_{a}^{2}} \cdot P_{1} G^{ \pm}\left(x, y, k_{a}\right)\right|_{y=0}\right)= \pm \sum_{p=1}^{n} \alpha_{p} \frac{a_{10}^{(1)} k_{20} C_{1}^{2} \sin ^{2}\left(d_{\mp} \nu_{1}\right) \nu_{1}^{2} \cos ^{2}\left(d_{ \pm} \nu_{1}\right)}{2 i \sqrt{\nu_{2}^{2}-\nu_{1}^{2}}} \rho \sin \theta \\
& \pm \sum_{p=1}^{n} \alpha_{p} \cdot \frac{a_{10}^{(1)} C_{2}^{2} \nu_{2} \sin ^{2}\left(d_{\mp} \nu\right) \cos \left(d_{ \pm} \nu\right)}{2} \cdot\left[\frac{1}{24} \cos \left(d_{ \pm} \nu_{2}\right) \nu_{2}^{3} \rho^{3} \sin 3 \theta \pm \sin \left(d_{ \pm} \nu\right) k_{20} \rho \cos \theta\right] \\
& \pm \sum_{p=1}^{n} \alpha_{p} \cdot a_{10}^{(1)} k_{20} g_{x}^{ \pm} \rho \sin \theta \mp a_{10}^{(1)} \alpha_{q} \frac{1}{\pi} k_{40} \rho^{-1} \sin \theta, \\
& a^{-3} M_{30}\left( \pm\left.\sqrt{\nu_{2}^{2}-k_{a}^{2}} \cdot a \cdot P_{2} G^{ \pm}\left(x, y, k_{a}\right)\right|_{y=0}\right)= \\
& \mp \sum_{p=1}^{n} \alpha_{p} \cdot \frac{a_{10}^{(2)} C_{2}^{2} \sin ^{2}\left(d_{\mp} \nu_{2}\right) \nu_{2}^{4} \cdot \sin ^{2}\left(d_{ \pm} \nu_{2}\right)}{8} \cdot \rho^{2} \cos 2 \theta \mp \frac{1}{\pi} \alpha_{q} k_{40} a_{10}^{(2)} \rho^{-2} \cos 2 \theta, \\
& a^{-3} M_{30}\left( \pm\left.\sqrt{\nu_{2}^{2}-k_{a}^{2}} \cdot a^{2} \cdot a_{10}^{(3)} D_{3} G^{ \pm}\left(x, y, k_{a}\right)\right|_{y=0}\right)= \\
& \pm \sum_{p=1}^{n} \alpha_{p} \cdot \frac{-a_{10}^{(3)} C_{2}^{2} \sin ^{2}\left(d_{\mp} \nu_{2}\right) \nu_{2}^{4} \cos ^{2}\left(d_{ \pm} \nu_{2}\right)}{2} \cdot \rho \sin \theta \pm a_{10}^{(3)} \alpha_{q} \frac{\nu_{2}^{2}}{2 \pi} k_{20} \rho^{-1} \sin \theta \pm a_{10}^{(3)} \alpha_{q} \frac{2}{\pi} k_{40} \rho^{-3} \sin 3 \theta \\
& a^{-3} M_{30}\left( \pm\left.\sqrt{\nu_{2}^{2}-k_{a}^{2}} \cdot a^{2} \cdot a_{20}^{(3)} D_{2} G^{ \pm}\left(x, y, k_{a}\right)\right|_{y=0}\right)= \\
& =\sum_{p=1}^{n} \alpha_{p} \cdot \frac{a_{20}^{(3)} C_{2}^{2} \sin ^{2}\left(d_{\mp} \nu_{2}\right) \nu_{2}^{3} \sin \left(d_{ \pm} \nu_{2}\right) \cos \left(d_{ \pm} \nu_{2}\right)}{2} \rho \sin \theta \mp \frac{1}{\pi} \alpha_{q} k_{30} a_{20}^{(3)} \rho^{-2} \cos 2 \theta \text {. }
\end{aligned}
$$

The harmonic summary for $f_{3}(z)=\frac{1}{2}\left(z^{3}+\frac{3}{2} \sqrt{z^{2}}+\left(\sqrt{z^{2}-1}\right)^{3}\right)$ can be presented as follows:

$$
\begin{aligned}
& \widehat{f}_{3}(z)=\sum_{q=1}^{n} \alpha_{q} \omega_{q}^{3} f_{3}\left(\frac{z-x_{q}}{\omega_{q}}\right)= \\
& \left\{\begin{array}{l}
\sum_{q=1}^{n} \alpha_{q} \omega_{q}^{3} \frac{z^{3}}{\omega_{q}^{3}}-\sum_{q=1}^{n} \alpha_{q} \omega_{q}^{3} \frac{3 x_{q} z^{2}}{\omega_{q}^{3}}+\sum_{q=1}^{n} \alpha_{q} \omega_{q}^{3} \frac{3 x_{q}^{2} z}{\omega_{q}^{3}}+\sum_{q=1}^{n} \alpha_{q} \omega_{q}^{3} \frac{3 \omega_{q}}{16 z}+\ldots, z \rightarrow+\infty, \\
-\sum_{q=1}^{n} \alpha_{q} \omega_{q}^{3} \frac{3 \omega_{q}}{16 z}-\ldots, z \rightarrow-\infty .
\end{array}\right.
\end{aligned}
$$

Here, we also obtain a set of positive degrees instead of one so we will need the same idea as for previous order. Separating summands from (8)-(11) with positive $\rho$ degrees, we can obtain the following representation for $\widehat{v}_{30}(\xi)$ :

$$
\begin{aligned}
& \widehat{v}_{30}(\xi)=\beta_{21} \widehat{X_{2}}(\xi)+\widetilde{\beta}_{21} \widehat{X_{2}}(\xi *)+\beta_{11} \widehat{X_{1}}(\xi)+\widetilde{\beta}_{11} \widehat{X_{1}}(\xi *)+ \\
& \beta_{32} \widehat{Y_{3}}(\xi)+\widetilde{\beta}_{32} \widehat{Y_{3}}(\xi *)+\beta_{22} \widehat{Y_{2}}(\xi)+\widetilde{\beta}_{22} \widehat{Y_{2}}(\xi *)+\beta_{12} \widehat{Y_{1}}(\xi)+\widetilde{\beta}_{12} \widehat{Y_{1}}(\xi *)
\end{aligned},
$$

where coefficients can be found:

$$
\begin{gathered}
\beta_{21}=\frac{-a_{10}^{(2)} C_{2}^{2} \sin ^{2}\left(d_{-} \nu_{2}\right) \nu_{2}^{4} \cdot \sin ^{2}\left(d_{+} \nu_{2}\right)}{8}, \quad \widetilde{\beta}_{21}=\frac{a_{10}^{(2)} C_{2}^{2} \sin ^{2}\left(d_{+} \nu_{2}\right) \nu_{2}^{4} \cdot \sin ^{2}\left(d_{-} \nu_{2}\right)}{8}, \\
\beta_{11}=\frac{a_{10}^{(1)} k_{20} C_{2}^{2} \nu_{2} \sin ^{2}\left(d_{-} \nu\right) \cos \left(d_{+} \nu\right) \sin \left(d_{+} \nu\right)}{2}+2\left(\frac{\sum_{q=1}^{n} \alpha_{q} x_{q}}{\sum_{q=1}^{n} \alpha_{q}}\right) \beta_{21},
\end{gathered}
$$




$$
\begin{aligned}
& \widetilde{\beta}_{11}=\frac{a_{10}^{(1)} k_{20} C_{2}^{2} \nu_{2} \sin ^{2}\left(d_{+} \nu\right) \cos \left(d_{-} \nu\right) \sin \left(d_{-} \nu\right)}{2}+2\left(\frac{\sum_{q=1}^{n} \alpha_{q} x_{q}}{\sum_{q=1}^{n} \alpha_{q}}\right) \widetilde{\beta}_{21}, \\
& \beta_{32}=\frac{a_{10}^{(1)} C_{2}^{2} \nu_{2}^{4} \sin ^{2}\left(d_{-} \nu_{2}\right) \cos ^{2}\left(d_{+} \nu_{2}\right)}{48}, \quad \widetilde{\beta}_{32}=\frac{a_{10}^{(1)} C_{2}^{2} \nu_{2}^{4} \sin ^{2}\left(d_{+} \nu_{2}\right) \cos ^{2}\left(d_{-} \nu_{2}\right)}{48} \\
& \beta_{22}=3\left(\frac{\sum_{q=1}^{n} \alpha_{q} x_{q}}{\sum_{q=1}^{n} \alpha_{q}}\right) \beta_{32}, \quad \widetilde{\beta}_{22}=3\left(\frac{\sum_{q=1}^{n} \alpha_{q} x_{q}}{\sum_{q=1}^{n} \alpha_{q}}\right) \widetilde{\beta}_{32} \\
& \beta_{12}=\frac{a_{10}^{(1)} k_{20} C_{1}^{2} \sin ^{2}\left(d_{-} \nu_{1}\right) \nu_{1}^{2} \cos ^{2}\left(d_{+} \nu_{1}\right)}{2 i \sqrt{\nu_{2}^{2}-\nu_{1}^{2}}}+a_{10}^{(1)} k_{20} g_{x}^{+}-3\left(\frac{\sum_{q=1}^{n} \alpha_{q} x_{q}^{2}}{\sum_{q=1}^{n} \alpha_{q}}\right) \beta_{32}+2\left(\frac{\sum_{q=1}^{n} \alpha_{q} x_{q}}{\sum_{q=1}^{n} \alpha_{q}}\right) \beta_{22} \\
& -\frac{a_{10}^{(3)} C_{2}^{2} \sin ^{2}\left(d_{-} \nu_{2}\right) \nu_{2}^{4} \cos ^{2}\left(d_{+} \nu_{2}\right)}{2}+\frac{a_{20}^{(3)} C_{2}^{2} \sin ^{2}\left(d_{-} \nu_{2}\right) \nu_{2}^{3} \sin \left(d_{+} \nu_{2}\right) \cos \left(d_{+} \nu_{2}\right)}{2} \\
& \widetilde{\beta}_{12}=\frac{a_{10}^{(1)} k_{20} C_{1}^{2} \sin ^{2}\left(d_{+} \nu_{1}\right) \nu_{1}^{2} \cos ^{2}\left(d_{-} \nu_{1}\right)}{2 i \sqrt{\nu_{2}^{2}-\nu_{1}^{2}}}+a_{10}^{(1)} k_{20} g_{x}^{-}-3\left(\frac{\sum_{q=1}^{n} \alpha_{q} x_{q}^{2}}{\sum_{q=1}^{n} \alpha_{q}}\right) \widetilde{\beta}_{32}+2\left(\frac{\sum_{q=1}^{n} \alpha_{q} x_{q}}{\sum_{q=1}^{n} \alpha_{q}}\right) \widetilde{\beta}_{22} \\
& -\frac{a_{10}^{(3)} C_{2}^{2} \sin ^{2}\left(d_{+} \nu_{2}\right) \nu_{2}^{4} \cos ^{2}\left(d_{-} \nu_{2}\right)}{2}-\frac{a_{20}^{(3)} C_{2}^{2} \sin ^{2}\left(d_{+} \nu_{2}\right) \nu_{2}^{3} \sin \left(d_{-} \nu_{2}\right) \cos \left(d_{-} \nu_{2}\right)}{2} \text {. }
\end{aligned}
$$

Particular solution of inhomogeneous equation can be obtained by integrating:

$$
\begin{aligned}
& v_{10}(\xi)=\frac{a_{10}^{(1)} C_{2}^{2} \nu_{2}^{2} \sin ^{2}\left(d_{-} \nu_{2}\right) \cos ^{2}\left(d_{+} \nu_{2}\right)}{2} \cdot \widehat{Y_{1}}(\xi)+\frac{a_{10}^{(1)} C_{2}^{2} \nu_{2}^{2} \sin ^{2}\left(d_{+} \nu_{2}\right) \cos ^{2}\left(d_{-} \nu_{2}\right)}{2} \cdot \widehat{Y_{1}}\left(\xi^{*}\right), \\
& \widetilde{v}_{30}(\xi)=-\nu_{2}^{2} \frac{a_{10}^{(1)} C_{1}^{2} \nu^{2} \sin ^{2}\left(d_{-} \nu\right) \cos ^{2}\left(d_{+} \nu\right)}{2} \\
& \times\left\{\left(\sum_{q=1}^{n} \alpha_{q}\right) \frac{1}{8} \rho^{3} \sin \theta+\left(\sum_{q=1}^{n} \alpha_{q} \frac{\omega_{q}^{2}}{8}\right) \rho \ln \rho \sin \theta-\frac{1}{8}\left(\sum_{q=1}^{n} \alpha_{q}\left(\frac{\omega_{q}^{2} x_{q}^{2}}{4}+\frac{\omega_{q}^{4}}{16}\right)\right) \rho^{-1} \sin 3 \theta-\ldots, \quad \xi_{2}>0,\right. \\
& \times\left\{\begin{array}{l}
-\left(\sum_{q=1}^{n} \alpha_{q} \frac{\omega_{q}^{2}}{8}\right) \rho \ln \rho \sin \theta+\frac{1}{8}\left(\sum_{q=1}^{n} \alpha_{q}\left(\frac{\omega_{q}^{2} x_{q}^{2}}{4}+\frac{\omega_{q}^{4}}{16}\right)\right) \rho^{-1} \sin 3 \theta+\ldots, \quad \xi_{2}<0,
\end{array}\right. \\
& -\nu_{2}^{2} \frac{a_{10}^{(1)} C_{2}^{2} \nu_{2}^{2} \sin ^{2}\left(d_{+} \nu_{2}\right) \cos ^{2}\left(d_{-} \nu_{2}\right)}{2} \\
& \times\left\{\begin{array}{l}
\left(\sum_{q=1}^{n} \alpha_{q} \frac{\omega_{q}^{2}}{8}\right) \rho \ln \rho \sin \theta-\frac{1}{8}\left(\sum_{q=1}^{n} \alpha_{q}\left(\frac{\omega_{q}^{2} x_{q}^{2}}{4}+\frac{\omega_{q}^{4}}{16}\right)\right) \rho^{-1} \sin 3 \theta-\ldots, \quad \xi_{2}>0, \\
-\left(\sum_{q=1}^{n} \alpha_{q}\right) \frac{1}{8} \rho^{3} \sin \theta-\left(\sum_{q=1}^{n} \alpha_{q} \frac{\omega_{q}^{2}}{8}\right) \rho \ln \rho \sin \theta+\frac{1}{8}\left(\sum_{q=1}^{n} \alpha_{q}\left(\frac{\omega_{q}^{2} x_{q}^{2}}{4}+\frac{\omega_{q}^{4}}{16}\right)\right) \rho^{-1} \sin 3 \theta+\ldots, \quad \xi_{2}<0 .
\end{array}\right.
\end{aligned}
$$


Finally, we obtain full solution for $\xi_{2}>0$ :

$$
\begin{aligned}
& v_{30}(\xi)=\beta_{21} \rho^{2} \cos 2 \theta+\beta_{11} \rho \cos \theta+\beta_{32} \rho^{3} \sin 3 \theta+\beta_{22} \rho^{2} \sin 2 \theta+\beta_{12} \rho \sin \theta \\
& -\nu_{2}^{2} \frac{a_{10}^{(1)} C_{2}^{2} \nu_{2}^{2} \sin ^{2}\left(d_{-} \nu_{2}\right) \cos ^{2}\left(d_{+} \nu_{2}\right)}{2} \times\left[\begin{array}{c}
\left(\sum_{q=1}^{n} \alpha_{q}\right) \frac{1}{8} \rho^{3} \sin \theta+\left(\sum_{q=1}^{n} \alpha_{q} \frac{\omega_{q}^{2}}{8}\right) \rho \ln \rho \sin \theta \\
-\frac{1}{8}\left(\sum_{q=1}^{n} \alpha_{q}\left(\frac{\omega_{q}^{2} x_{q}^{2}}{4}+\frac{\omega_{q}^{4}}{16}\right)\right) \rho^{-1} \sin 3 \theta-\ldots
\end{array}\right] \\
& -\nu_{2}^{2} \frac{a_{10}^{(1)} C_{2}^{2} \nu_{2}^{2} \sin ^{2}\left(d_{+} \nu_{2}\right) \cos ^{2}\left(d_{-} \nu_{2}\right)}{2} \cdot\left[\begin{array}{c}
\left(\sum_{q=1}^{n} \alpha_{q} \frac{\omega_{q}^{2}}{8}\right) \rho \ln \rho \sin \theta \\
-\frac{1}{8}\left(\sum_{q=1}^{n} \alpha_{q}\left(\frac{\omega_{q}^{2} x_{q}^{2}}{4}+\frac{\omega_{q}^{4}}{16}\right)\right) \rho^{-1} \sin 3 \theta-\ldots
\end{array}\right] \\
& +\sum_{j=1}^{\infty}\left(\beta_{12} b_{1 j}^{+}+\widetilde{\beta}_{12} b_{1 j}^{-}+\beta_{22} b_{2 j}^{+}+\widetilde{\beta}_{22} b_{2 j}^{-}+\beta_{32} b_{3 j}^{+}+\widetilde{\beta}_{32} b_{3 j}^{-}\right) \rho^{-j} \sin j \theta \\
& +\sum_{j=1}^{\infty}\left(\beta_{11} a_{1 j}^{+}-\widetilde{\beta}_{11} a_{1 j}^{-}+\beta_{21} a_{2 j}^{+}+\widetilde{\beta}_{21} a_{2 j}^{-}\right) \rho^{-j} \cos j \theta .
\end{aligned}
$$

For $\xi_{2}<0$, one has:

$$
\begin{aligned}
& v_{30}(\xi)=\widetilde{\beta}_{21} \rho^{2} \cos 2 \theta+\widetilde{\beta}_{11} \rho \cos \theta-\widetilde{\beta}_{32} \rho^{3} \sin 3 \theta-\widetilde{\beta}_{22} \rho^{2} \sin 2 \theta-\widetilde{\beta}_{12} \rho \sin \theta \\
& -\nu_{2}^{2} \frac{a_{10}^{(1)} C_{2}^{2} \nu_{2}^{2} \sin ^{2}\left(d_{-} \nu_{2}\right) \cos ^{2}\left(d_{+} \nu_{2}\right)}{2} \times\left[\begin{array}{l}
-\left(\sum_{q=1}^{n} \alpha_{q} \frac{\omega_{q}^{2}}{8}\right) \rho \ln \rho \sin \theta+ \\
+\frac{1}{8}\left(\sum_{q=1}^{n} \alpha_{q}\left(\frac{\omega_{q}^{2} x_{q}^{2}}{4}+\frac{\omega_{q}^{4}}{16}\right)\right) \rho^{-1} \sin 3 \theta+\ldots
\end{array}\right] \\
& -\nu_{2}^{2} \frac{a_{10}^{(1)} C_{2}^{2} \nu_{2}^{2} \sin ^{2}\left(d_{+} \nu_{2}\right) \cos ^{2}\left(d_{-} \nu_{2}\right)}{2} \times\left[\begin{array}{l}
-\left(\sum_{q=1}^{n} \alpha_{q}\right) \frac{1}{8} \rho^{3} \sin \theta-\left(\sum_{q=1}^{n} \alpha_{q} \frac{\omega_{q}^{2}}{8}\right) \rho \ln \rho \sin \theta+ \\
+\frac{1}{8}\left(\sum_{q=1}^{n} \alpha_{q}\left(\frac{\omega_{q}^{2} x_{q}^{2}}{4}+\frac{\omega_{q}^{4}}{16}\right)\right) \rho^{-1} \sin 3 \theta+\ldots
\end{array}\right] \\
& +\sum_{j=1}^{\infty}\left(-\beta_{12} b_{1 j}^{+}-\widetilde{\beta}_{12} b_{1 j}^{-}-\beta_{22} b_{2 j}^{+}-\widetilde{\beta}_{22} b_{2 j}^{-}-\beta_{32} b_{3 j}^{+}-\widetilde{\beta}_{32} b_{3 j}^{-}\right) \rho^{-j} \sin j \theta \\
& +\sum_{j=1}^{\infty}\left(\beta_{11} a_{1 j}^{+}-\widetilde{\beta}_{11} a_{1 j}^{-}+\beta_{21} a_{2 j}^{+}+\widetilde{\beta}_{21} a_{2 j}^{-}\right) \rho^{-j} \cos j \theta .
\end{aligned}
$$

Matching terms of order $\rho^{-1} \sin \theta$, one obtains:

$$
\begin{aligned}
& \mp a_{10}^{(1)} \alpha_{q} \frac{1}{\pi} k_{40} \pm a_{10}^{(3)} \alpha_{q} \frac{\nu_{2}^{2}}{2 \pi} k_{20}= \\
& \pm \nu_{2}^{2} \frac{a_{10}^{(1)} C_{2}^{2} \nu_{2}^{2}\left[\sin ^{2}\left(d_{-} \nu_{2}\right) \cos ^{2}\left(d_{+} \nu_{2}\right)+\sin ^{2}\left(d_{+} \nu_{2}\right) \cos ^{2}\left(d_{-} \nu_{2}\right)\right]}{2} \cdot\left[\frac{3}{8}\left(\sum_{p=1}^{n} \alpha_{p}\left(\frac{\omega_{p}^{2} x_{p}^{2}}{4}+\frac{\omega_{p}^{4}}{16}\right)\right)\right] \\
& \pm\left(\beta_{12} b_{11}^{+}+\widetilde{\beta}_{12} b_{11}^{-}+\beta_{22} b_{21}^{+}+\widetilde{\beta}_{22} b_{21}^{-}+\beta_{32} b_{31}^{+}+\widetilde{\beta}_{32} b_{31}^{-}\right) \Rightarrow \\
& -a_{10}^{(1)} \alpha_{q} \frac{1}{\pi} k_{40}+a_{10}^{(3)} \alpha_{q} \frac{\nu_{2}^{2}}{2 \pi} k_{20}=\nu_{2}^{2} \frac{3 a_{10}^{(1)} C_{2}^{2} \nu_{2}^{2} S_{+}}{16} \cdot\left(\sum_{p=1}^{n} \alpha_{p}\left(\frac{\omega_{p}^{2} x_{p}^{2}}{4}+\frac{\omega_{p}^{4}}{16}\right)\right)-\left(\sum_{p=1}^{n} \alpha_{p} \frac{3 \omega_{p}^{4}}{16} \cdot \frac{a_{10}^{(1)} C_{2}^{2} \nu_{2}^{4} S_{+}}{48}\right)
\end{aligned}
$$

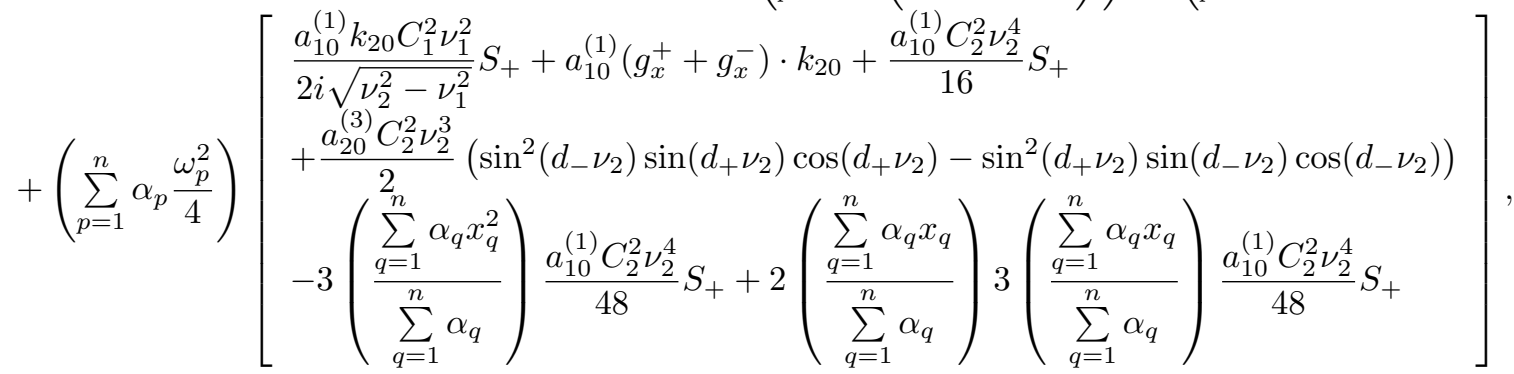

where $S_{ \pm}=\sin ^{2}\left(d_{-} \nu_{2}\right) \cos ^{2}\left(d_{+} \nu_{2}\right) \pm \sin ^{2}\left(d_{+} \nu_{2}\right) \cos ^{2}\left(d_{-} \nu_{2}\right)$. 
As we can see this system of equations is not linear because of expressions like $\left(\frac{\sum_{p=1}^{n} \alpha_{p} x_{p}^{2}}{\sum_{p=1}^{n} \alpha_{p}}\right)$. That's why we will consider only imaginary part of both sides and it will make system actually linear:

$$
\begin{gathered}
-a_{10}^{(1)} \alpha_{q} \frac{1}{\pi} \operatorname{Im} k_{40}=\left(\sum_{p=1}^{n} \alpha_{p} \frac{\omega_{p}^{2}}{4}\right)\left[-\frac{a_{10}^{(1)} k_{20} C_{1}^{2} \nu_{1}^{2}}{2 \sqrt{\nu_{2}^{2}-\nu_{1}^{2}}}\left(\sin ^{2}\left(d_{-} \nu_{1}\right) \cos ^{2}\left(d_{+} \nu_{1}\right)+\sin ^{2}\left(d_{+} \nu_{1}\right) \cos ^{2}\left(d_{-} \nu_{1}\right)\right)\right] \Rightarrow \\
\beta \alpha_{q}=\sum_{p=1}^{n} \alpha_{p} \frac{\omega_{p}^{2}}{4}, \quad \beta=\frac{2 \sqrt{\nu_{2}^{2}-\nu_{1}^{2}} \operatorname{Im} k_{40}}{\pi k_{20} C_{1}^{2} \nu_{1}^{2}\left(\sin ^{2}\left(d_{-} \nu_{1}\right) \cos ^{2}\left(d_{+} \nu_{1}\right)+\sin ^{2}\left(d_{+} \nu_{1}\right) \cos ^{2}\left(d_{-} \nu_{1}\right)\right)} \Rightarrow \\
\sum_{q=1}^{n} c_{\omega_{q}}=\frac{2 \sqrt{\nu_{2}^{2}-\nu_{1}^{2}} \operatorname{Im} k_{40}}{\pi k_{20} C_{1}^{2} \nu_{1}^{2}\left(\sin ^{2}\left(d_{-} \nu_{1}\right) \cos ^{2}\left(d_{+} \nu_{1}\right)+\sin ^{2}\left(d_{+} \nu_{1}\right) \cos ^{2}\left(d_{-} \nu_{1}\right)\right)} \Rightarrow \\
\operatorname{Im} k_{40}=\frac{-\pi^{2} C_{1}^{2} \nu_{1}^{2} C_{2}^{2} \nu_{2}^{2}\left(1-\cos \left(2 d_{-} \nu_{1}\right) \cos \left(2 d_{+} \nu_{1}\right)\right)\left(1-\cos \left(2 d_{-} \nu_{2}\right) \cos \left(2 d_{+} \nu_{2}\right)\right)}{8 \sqrt{\nu_{2}^{2}-\nu_{1}^{2}}}\left(\sum_{q=1}^{n} c_{\omega_{q}}\right)^{2}
\end{gathered}
$$

We can compare this formula with result from single-window case:

$$
\operatorname{Im} k_{40}=\frac{-\pi^{2} C_{1}^{2} \nu_{1}^{2} C_{2}^{2} \nu_{2}^{2}}{128 \sqrt{\nu_{2}^{2}-\nu_{1}^{2}}}\left(1-\cos \left(2 d_{-} \nu_{1}\right) \cos \left(2 d_{+} \nu_{1}\right)\right)\left(1-\cos \left(2 d_{-} \nu_{2}\right) \cos \left(2 d_{+} \nu_{2}\right)\right)
$$

and verify it is absolutely consistent, the difference is just in multiplier $\left(\sum_{q=1}^{n} c_{\omega_{q}}\right)^{2}$ characterizing common size of windows. As usual, it is the square of the coefficient appearing in the first order term.

\section{Conclusion}

The results for the imaginary part of the resonance allow us to pose the problem of minimizing/maximizing the lifetime of the resonance by changing the configuration of the windows, e.g., fixing the summary size of windows. The results pertaining to the real part of the resonance, give one an estimation of the shift of resonance with respect to the threshold. These results can be useful for the description of "quantum waveguide - quantum dot - quantum waveguide" systems. One can find such systems in different nanotechnology applications.

\section{References}

[1] Lord Rayleigh O.M. The theory of Helmholtz Resonator. Proceeding of Royal Society, 1916, 638, P. $265-275$.

[2] Morse F.M. Feshbach G. Methods of Theoretical Physics, V. 2. Foreign Literature Publishing House, 1960,986 p.

[3] Kiselev A.A., Pavlov B.S. The eigenfrequencies and eigenfunctions of the Laplace operator of the Neumann problem in a system of two coupled resonators. Theor. Math. Phys., 1994, 100(3), P. 354-366.

[4] Gadylshin R.R. The existence and asymptotics of poles with a small imaginary part for the Helmholtz resonator. Uspekhi of Mathematical Sciences, 1997, 52(313), P. 71-72.

[5] Borisov D.I. Discrete spectrum of an asymmetric pair of waveguides coupled through a window. Sb. Math., 2006, 197(4), P. 475-504.

[6] Achilleos V., Richoux O., et.al. Acoustic solitons in waveguides with Helmholtz resonators: Transmission line approach. Phys.Rev. E., 2015, 91, P. 023204.

[7] Martinez A., Nedelec L. Optimal lower bound of the resonance widths for a Helmoltz tube-shaped resonator. J. Spectral Th., 2012, 2, P. 203223.

[8] Gadyl'shin R.R. A two-dimensional analogue of the Helmholtz resonator with ideally rigid walls. Diff. Uravn., 1994, 30(2), P. 221-229. Translation in: Diff. Eq., 1994, 30(2), P. 201-209.

[9] Gadyl'shin R.R. Influence of the position of the opening and its shape on the properties of a Helmholtz resonator. Theor. Math. Phys., 1992, 93, P. 1151-1159.

[10] Borisov D., Exner P. Distant perturbation asymptotics in window-coupled waveguides. I. The non-threshold case. J. Math. Phys., 2006, 47(10), P. 113502(1-24).

[11] Popov I. Asymptotics of bound states and bands for laterally coupled waveguides and layers. J. Math. Phys., 2002,43 , P. $215-234$.

[12] Borisov D.I., Gadyl'shin R.R. On the spectrum of the Laplacian with frequently alternating boundary conditions. Theor. Math. Phys., 1999, 118(3), P. 272-277.

[13] Briet Ph., Dittrich J., Soccorsi E. Scattering through a straight quantum waveguide with combined boundary conditions. J. Math. Phys., 2014, 55, P. 112104.

[14] Ilyin A.M. Matching of the asymptotic expansions of solutions. Science, Moscow, 1989, $336 \mathrm{p}$.

[15] Exner P., Kreicirik D. Waveguides coupled through a semitransparent barrier: a Birman-Schwinger analysis. Rev. Math. Phys., 2001, 13, P. 307-334.

[16] Exner P., Kreicirik D. Quantum waveguides with a lateral semitransparent barrier: spectral and scattering properties. J. Phys. A, 1999, 32, P. 4475. 
[17] Popov I.Yu. The extension theory, domains with semitransparent surface and the model of quantum dot. Proc. Royal Soc. London A., 1996, 452(1950), P. 1505-1515.

[18] Popov I.Y. Model of a quantum point as a cavity with semitransparent boundary. Physics of the Solid State, 1994, 36(7), P. 1918-1922.

[19] Popov I.Yu. The extension theory and the opening in semitransparent surface. J. Math. Phys., 1992. 33(5), P. $1685-1689$.

[20] Ikebe T., Shimada S. Spectral and scattering theory for the Scrodinger operator with penetrable wall potentials. J.Math. Kyoto Univ., 1991, 31(1), P. 219-258.

[21] Popov I.Y., Trifanova E.S., Vorobiev A.M. 2D waveguides asymptotics of eigenvalue induced by a window in a semitransparent separating wall. Bulletin of the Transilvania University of Brasov, Series III: Mathematics, Informatics, Physics, 2021, 63(1), P. 221-230.

[22] Vorobiev A.M., Popov I.Y. Resonances in two-dimensional quantum waveguides separated by semitransparence wall with small window. Technical Physics Letters, 2021, 47(9), P. 847-849.

[23] Exner P., Kondej S., Lotoreichik V. Asymptotics of the bound state induced by delta-interaction supported on a weakly deformed plane. $J$. Math. Phys., 2018, 59, P. 013051.

[24] Behrndt J., Exner P., et.al. Approximation of Schroedinger operators with delta-interactions supported on hypersurfaces. Math. Nachr., 2017, 290, P. 12151248.

[25] Popov I.Yu. The operator extension theory, semitransparent surface and short range potential. Math. Proc. Cambridge Phil. Soc., 1995, 118, P. 555-563.

[26] Tikhonov A.N., Samarskii A.A. Equations of Mathematical Physics. M.: Science, 1972, 531 p. 